\title{
Test of CCD Precision Limits for Differential Photometry
}

\author{
L. B. ROBINSON AND M. Z. WEI \\ UCO/Lick Observatory, University of California, Santa Cruz, California 95064 \\ W. J. BORUCKI AND E. W. DUNhaM \\ NASA AMES Research Center, MS 245-6, Moffett Field, California 94035 \\ C. H. FORD AND A. F. GRANAdOS \\ SETI Institute, 2035 Landings Drive, Mt. View, California 94043 \\ Received 1995 June 1; accepted 1995 July 31
}

\begin{abstract}
Results of tests to demonstrate the very high differential-photometric stability of CCD light sensors are presented. The measurements reported here demonstrate that in a controlled laboratory environment, a front-illuminated $\mathrm{CCD}$ can provide differential-photometric measurements with reproducible precision approaching one part in $10^{5}$. Practical limitations to the precision of differential-photometric measurements with CCDs and implications for spaceborne applications are discussed.
\end{abstract}

\section{INTRODUCTION}

Even though CCDs have now superseded other light sensors for most astronomical photometry, there is widespread uncertainty as to their attainable precision. Limitations produced by external factors are sometimes thought to be intrinsic limitations of the devices themselves, giving unnecessarily pessimistic predictions of what is possible with $\mathrm{CCD}$ sensors. Earlier work bearing on this subject includes papers by Buffington et al. (1990, 1991), Gilliland et al. (1988, 1991), and Young et al. (1991).

The measurements reported here demonstrate that under ideal circumstances, a front-illuminated $\mathrm{CCD}$ can provide differential-photometric measurements with reproducibility approaching one part in $10^{5}$. Although photometric precision for astronomy is normally much poorer due to factors such as the limited number of photons available and atmospheric scintillation, it is important to understand how to separate the limitations produced by the detector from limitations due to external and possibly correctable factors.

The work reported here was prompted by a proposal to detect planets orbiting other stars by observing transits of the planets across the stellar disks. The transits would be detected by making precise measurements of photometric variations for a large number of stars over an extended period of time from a space platform, where many of the problems seen by ground-based observers can be avoided. This paper describes the experimental tests of one $\mathrm{CCD}$, then discusses how the results were analyzed and how they may be interpreted. In addition, possible sources of photometric error not measured by this experiment are mentioned.

\section{EXPERIMENTAL DETAILS}

In order to demonstrate that precise measurements are possible with a $\mathrm{CCD}$, images in the laboratory were taken of an artificial star-field over a ten-day period. The goal of the experiment was to show that one can detect small temporal changes in the light level of any one star when compared to the average of the other stars in the field.

The experiment was carried out at the Lick Observatory
CCD laboratory using a front-illuminated Reticon $512 \times 512$ CCD with 27 micron pixels. The device was operated in multipinned-phase (MPP) mode at a stabilized temperature of about $-110^{\circ} \mathrm{C}$, and had a full well of approximately $3 \times 10^{5}$ electrons per pixel. The output signal from the CCD was attenuated to produce about 40 electrons per DN, and signal levels of about $6000 \mathrm{DN}$ were used to avoid saturating the CCD. A 16 bit analog-to-digital converter (ADC) was used for the measurements. The CCD had good chargetransfer characteristics for both large and small signals, although there were a few low-level charge traps scattered over the detector's sensitive area. In order to reduce the effects of photon shot-noise, 100 successive exposures were added together for each recorded image, resulting in approximately $40 \times 6000 \times 100=24 \times 10^{6}$ electrons per pixel in the final images.

The apparatus consisted of the CCD mounted in a liquidnitrogen-cooled dewar, with a simple illumination system to produce artificial star images. The CCD position was referenced to the front face of the dewar, rather than to the liquidnitrogen cold surface, to reduce mechanical motion as the coolant evaporated. The CCD was illuminated with light from a single red LED that passed through two separated translucent plastic diffusers, then through a mask consisting of a metal plate drilled with holes of five different sizes. The mask was imaged onto the CCD by a Canon $f / 1.8$ lens. The intensity of illumination across the field was uniform to within about $30 \%$. In order to avoid jarring and other errors that might be caused by a mechanical shutter, the exposures were controlled by switching the LED on and off with a relay. The assembly was mounted with the optical axis vertical and placed in a large enclosure whose temperature was controlled to within about $0.1^{\circ} \mathrm{C}$. Metal rods were used to clamp the optical assembly to the dewar while cylinders of PVC with 1 in. thick walls were used as spacers for the optical setup.

The liquid-nitrogen-cooled dewar had a hold time of only about $20 \mathrm{~h}$, so regular refilling was required during the 10 day run reported here. As will be noted from the data presented later, the $\mathrm{LN}$ refill was a major cause of mechanical 


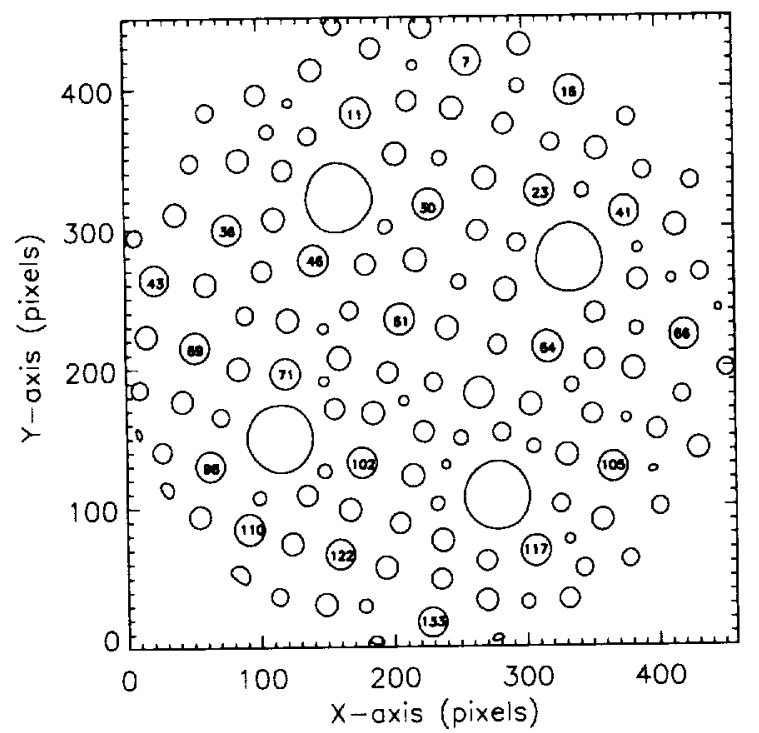

FIG. 1-This shows the size and distribution of artificial stars imaged on the CCD. Only data from the marked medium-size "stars" were analyzed for this report.

instability. In order to limit this problem, the fill funnel was carefully fastened in place and isolated as well as possible from the rest of the equipment. The temperature-dependent resistance of a carbon resistor mounted inside the dewar allowed a rough estimate of the $\mathrm{LN}$ level, to allow refilling without overflow.

\section{EXPERIMENTAL RESULTS}

Although a number of preliminary test runs were carried out in which various problems with the experimental setup were discovered and corrected, only data from the final 10 day run are presented here. Frames of $451 \times 460$ pixels were taken repeatedly and the average of each succeeding 100 frames was recorded as a single image; each recorded image represents about 35-min worth of data. Data were recorded continuously over the 10-day period, producing 354 recorded images. In order to reach an arbitrary shot-noise limit of 1 part in $10^{5}$ it was necessary to add the data from about 400 pixels, so "stars" of about that size were chosen for the analysis. The "star field" is shown in Fig. 1 and the cross section of a typical "star" used in the analysis is shown in Fig. 2.

To correct for small unavoidable variations of the light intensity and amplifier gain, data from each star were divided by the average signal level from all the stars. Raw data and normalized data from a typical star are shown in Figs. 3 and 4. Note the sudden changes when the dewar was refilled. The large spike in the normalized brightness following image 50 (Fig. 4) corresponds to a single instance when the dewar was overfilled, resulting in spillage that produced thermally induced image motion. The motion of the centroid of the same star is shown in Fig. 5(a). Although the LED was driven by a voltage-regulated power supply, a $0.5 \%$ variation of average raw signal level is seen, much of it following a diurnal pattern. The diurnal variation may be explained by the fact

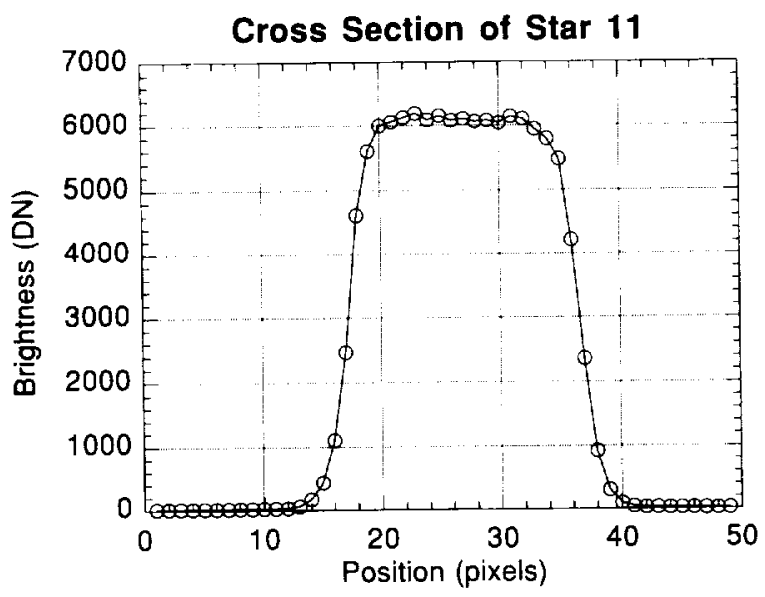

FIG. 2-A cross section of a typical star gives an indication of the sharpness and focus of the images. No change in focus or image sharpness was detected during the 10-day run.

that only the $\mathrm{CCD}$ analog-clock drivers and the preamp were in the temperature-controlled room with the dewar and CCD. The ADC and other electronics were subject to daily temperature variations of 2 to $5^{\circ} \mathrm{F}$.

\section{DATA REDUCTION}

A square $28 \times 28$ pixel aperture was used to extract the star data. A larger aperture was not used due to crowding of the artificial stars. No background subtraction was performed because most of the background light was actually scattered light from the stars themselves. (Background amounted to about $0.5 \%$ of the total signal.) The total $1 \sigma$ fractional error (photon noise plus detector noise) was $5.0 \times 10^{-5}$ when averaged over the full data set after the data were normalized using the average signal level of all the stars. Part of this error is due to photon noise, which is at the level of $1.18 \times 10^{-5}$. We suspected that additional error would be due to small motions of the artificial stars across the nonuni-

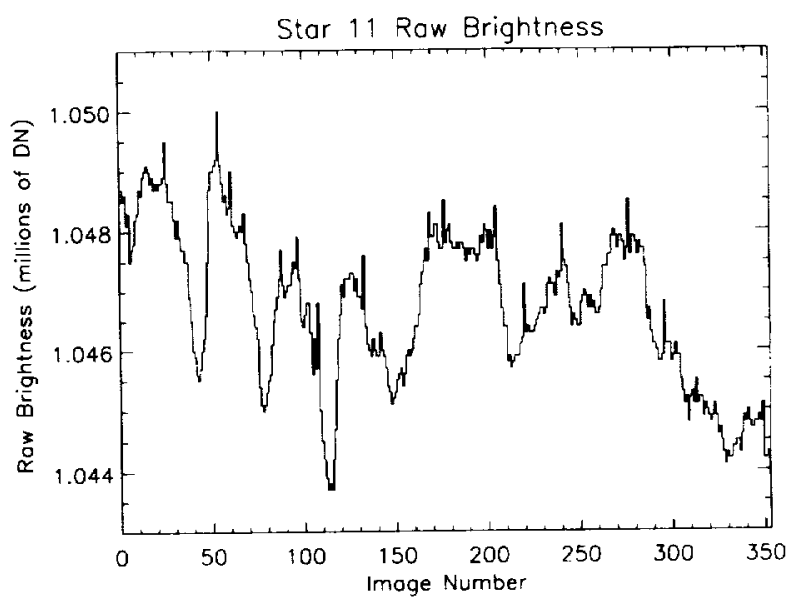

Fig. 3-The absolute signal level seen during the 10-day run varied by about $0.5 \%$. Causes of variation include mechanical motions and temperature variations due to regular refilling of the liquid-nitrogen dewar, as well as some diumal temperature variations that affected power supplies, amplifiers, and the analog-to-digital converter. 


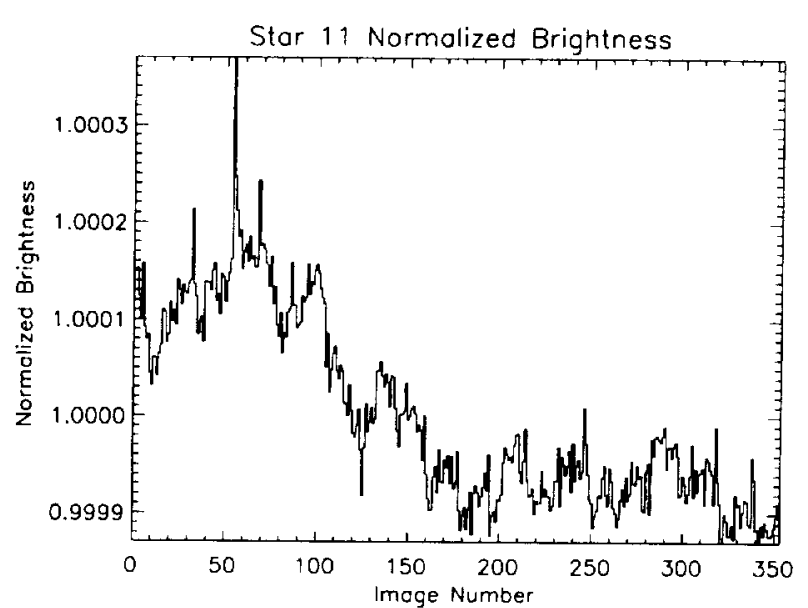

FIG. 4-After dividing the signal from "star" 11 by the average signal from all 21 tested "stars," the signal variation is reduced to roughly five parts in $10^{5}$. Note the improved stability during the second half of the run. This is believed mainly due to a drop in excess dark current during the first few days.

formly sensitive face of the CCD (Jorden et al. 1994), which would lead to reproducible errors that could be measured and corrected. To assess this possibility, the star images were individually centroided in each recorded image using marginal analysis (Elliot et al. 1989).

It is apparent that changes in the normalized brightness of a star are correlated to some extent with motions of its centroid. The periodic spike features seen in Fig. 4 correspond to the times when the dewar was refilled. Changes in the weight of the dewar, as well as local temperature changes due to the LN fill process, cause mechanical motion of the CCD and of the optical assembly. The resulting image motions [see Fig. 5 (a) for the motion of star 11] provide a well-sampled function of brightness versus centroid position. As expected, the polysilicon electrode structure on the front side of the CCD caused a well-defined correlation (or anticorrelation) between brightness and image position to be seen for most of the stars. This relationship is plotted in Fig. 5(b) for star 11, and it is seen that for this star, the $X$ position is correlated with brightness, while the $Y$ position is anticorrelated. In general, $X$ and $Y$ are either correlated or anticorrelated, depending on the image position with respect to the CCD structure. For the sample of 21 stars that were analyzed, correlation/anticorrelation occurred roughly an equal number of times for $X$ and $Y$. Of the four stars that showed little or no $X / Y$ correlation, one had the lowest uncorrected fractional error $\left(1.7 \times 10^{-5}\right)$, and the remaining three showed a weak dependence on raw brightness, probably due to nonlinear system response. Figure 6 shows star 43's behavior.

Because of the strong linear correlation/anticorrelation observed between normalized brightness and image position, as well as the observed weak dependence of normalized brightness on raw brightness, the normalized brightness data for each star were fit independently with a multipleparameter linear least-squares fit:

$$
B_{\mathrm{fit}}=C_{1}+C_{2} \delta X+C_{3} \delta Y+C_{4} \delta B .
$$

Here, $B_{\text {fit }}$ is the fitted value of the normalized brightness

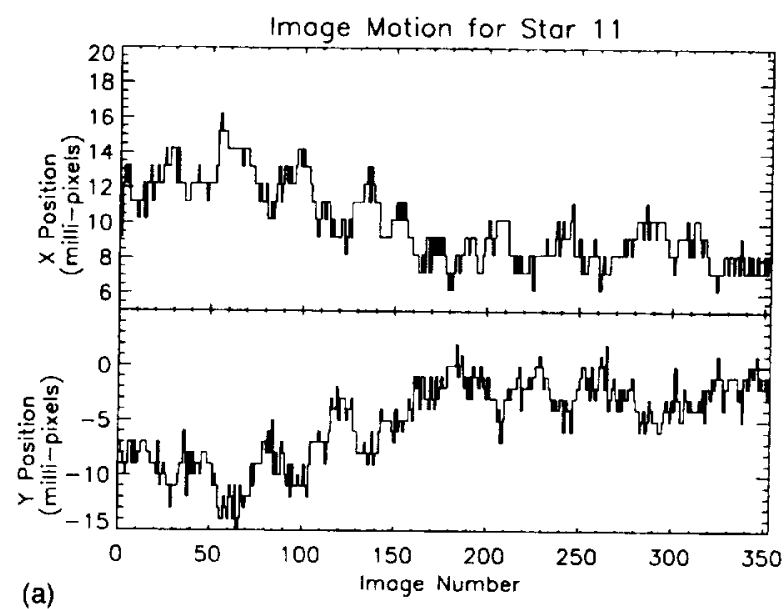

(a)
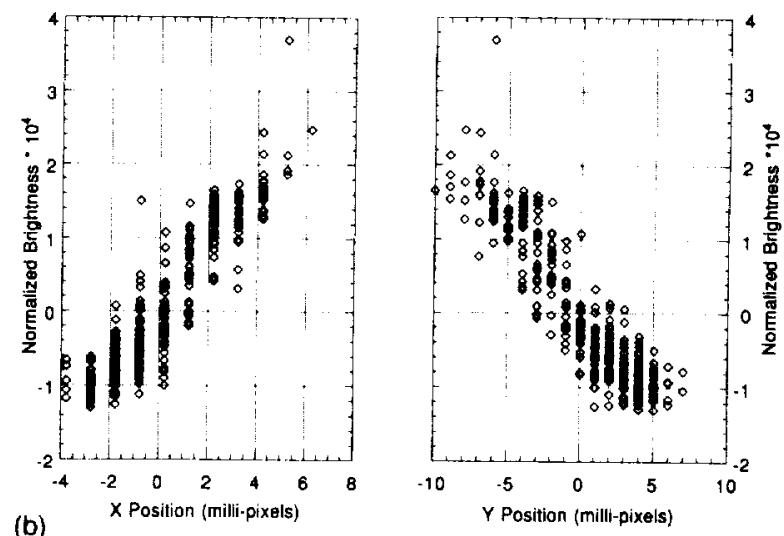

FIG. 5-(a) The centroids of the "stars" moved slightly on the CCD. The motions were somewhat repeatable; the main motion was closely synchronized with the periodic refilling of the dewar. (b) There is a clear correlation between the position of the "star" (here star 11) on the CCD and the normalized brightness. This is almost certainly due to the nonuniform sensitivity across each pixel, caused by light attenuation of the polysilicon electrodes that cover the $\mathrm{CCD}$.

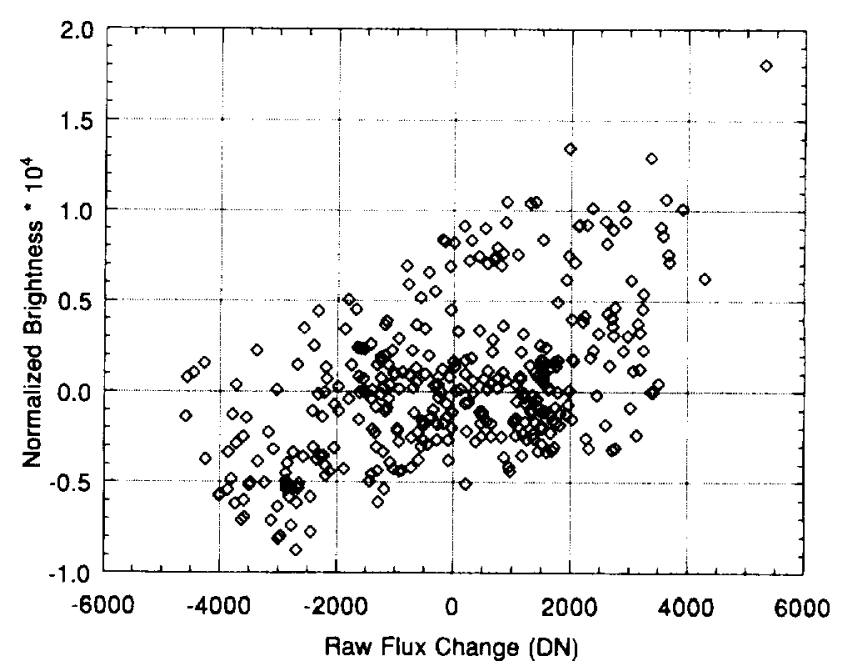

FiG. 6-There is a small correlation between the actual raw signal level and the normalized brightness. This is believed due to small nonlinearities in the electronic system. 


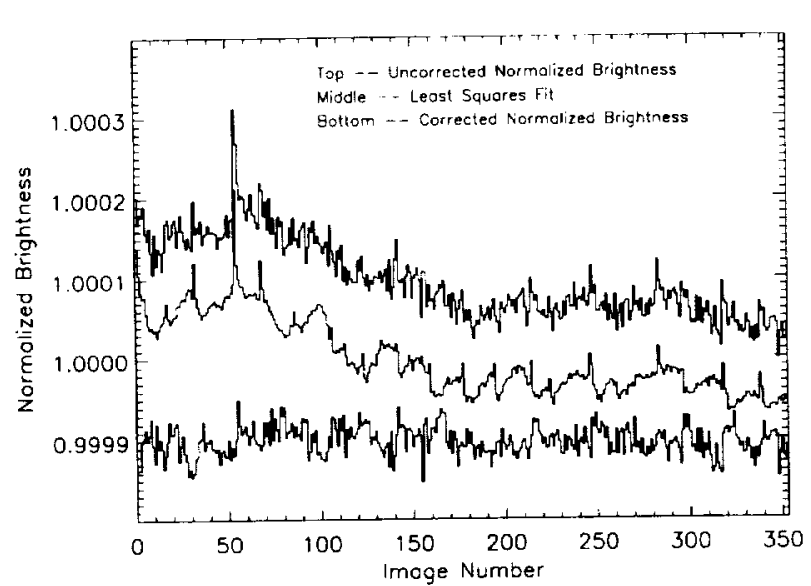

Fig. 7-By subtracting measured corrections due to changes in signal level and to motion of the image on the CCD, a considerably improved residual error is found, as shown by the lower curve. The upper curve shows uncorrected normalized data, and the middle curve shows the least-squares linear fit for this star (star number 7).

of the image in question, the $C_{i} \mathrm{~s}$ are fitted constants and $\delta X$, $\delta Y$, and $\delta B$ are the differences between the instantaneous values of the $X$ position, $Y$ position, and Brightness for a given star image minus the corresponding average value taken over the whole data set for that star.

The resulting fit for star 7 is plotted as the middle line in Fig. 7 with the normalized data plotted as the upper line for comparison. The difference between the data and the fit is plotted as the lower line in the figure. The fitting process provides about a factor of 3 improvement over the raw data, yielding a $1 \sigma$ fractional error of $1.8 \times 10^{-5}$ and does a good job of flattening the data and reducing the spikes caused by motion that occurred when the dewar was refilled, although the standard deviation is still above the shot-noise limit of $1.18 \times 10^{-5}$. Note however that the fractional error improved with time. The error in the corrected data for the first 120 hours of the test was $1.96 \times 10^{-5}$ and dropped to $1.61 \times 10^{-5}$ for the last 100 hours. The implication is that as the system stabilizes with time, we approach the shot-noise limit. Assuming that the data from the second half of the experiment are representative of a stable system, then by subtracting in quadrature the photon-noise fractional error of $1.18 \times 10^{-5}$ (as measured for this experiment) from the total measured fractional error of $1.6 \times 10^{-5}$, we find the fractional error contribution from the CCD plus any uncorrected experimental errors to be $1.1 \times 10^{-5}$. The result of fitting all stars over the whole duration of the experiment is shown in Fig. 8.

In order to determine the expected residual detectorrelated noise for the proposed long-term planetary detection observing program mentioned earlier, we tried using only the data from the last half of the test run, quadratically subtracted the photon-noise contribution, and averaged the data to an integration time of $5 \mathrm{hr}$. The result of this analysis is shown in Fig. 9. The $1 \sigma$ fractional error for all the stars in this case is $0.99 \times 10^{-5}$.

The larger errors during the first part of the experiment are not surprising, since the CCD was severely overexposed to light while cold, during the initial setup. This produced

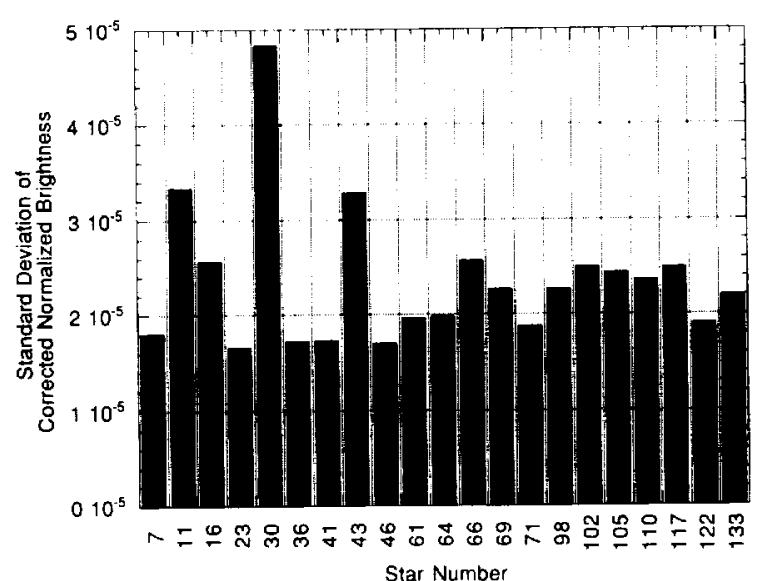

FIG. 8-The standard deviation of the corrected signal level for the 10-day run is approximately $1.8 \times 10^{-5}$. Larger deviation of a few "stars" may be due to local imperfections of the $C C D$. This error includes $1.18 \times 10^{-5}$ due to photon shot noise, and is at an integration time of $35 \mathrm{~min}$.

excess dark current whose decay could readily be detected during the first few days. The overflow and spillage of liquid nitrogen after the first day of operation may have also contributed uncorrected errors.

\section{DISCUSSION}

This experiment was designed to demonstrate the potential of CCDs for a specific photometric project where very small and relatively short-term variations in brightness are to be measured for a large number of stars, and the average brightness of all the stars can be used to remove most instrument-induced variations. Absolute photometry is not an issue, and the background level will be very small and quite constant. Since each star will illuminate its own set of pixels, nonuniform color response should not interfere with the measurements. Changes lasting a few hours are of major interest for the planned observations.

There may be some concern about the possible effects of cosmic-ray induced signals, as well as gradually increasing radiation damage to the $\mathrm{CCD}$. However, the rate of cosmic

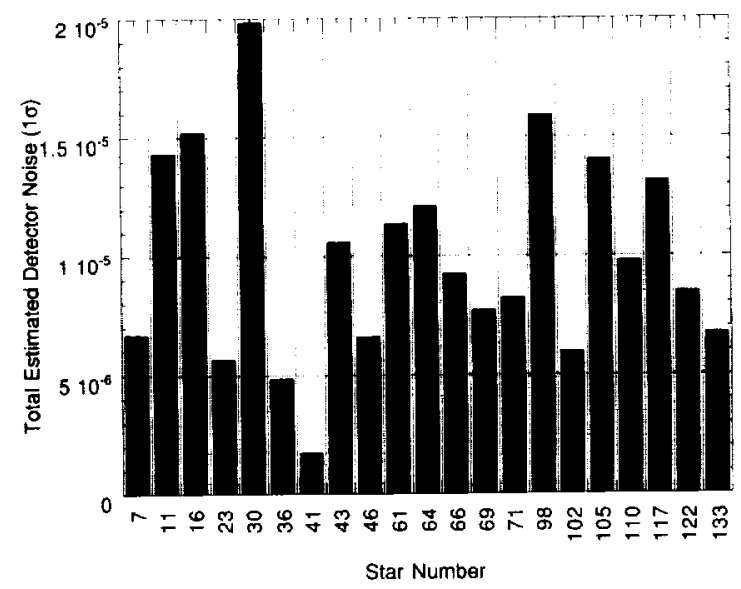

FIG. 9-The standard deviation can be further improved by using only the more stable data from the last half of the run, and by averaging over a 5 -hr interval, which is more representative of the planned experiment. 
rays in space should be about $1 \mathrm{~s}^{-1} \mathrm{~cm}^{-2}$. If each star covers 15 pixels, each 15 microns square, an area of about $3 \times 10^{-5}$ $\mathrm{cm}^{2}$, there would only be two or three hits per star per day, an effect very much smaller than shot-noise. Radiation damage may change the charge-transfer efficiency, and may also lead to increased dark signal, but these changes should easily be detected and corrected. Of course, permanent changes in local sensitivity due to radiation damage may be indistinguishable from permanent changes in the brightness of a star.

We recognize that there are many other limitations to photometric accuracy with CCDs, particularly for ground-based observations (see Young et al. 1991). While the data from this experiment demonstrate good intrinsic long-term stability for a front-illuminated CCD used for relative photometry at one color with a number of stars on the CCD, the experiment does not address numerous other problems seen in astronomical photometry.

One noteworthy problem is that the spatial response of most CCDs is wavelength dependent. This is well known for back-side-illuminated CCDs, where the UV and blue response can vary dramatically over the surface of a CCD, and also may change over a short time for CCDs whose UV response is obtained by UV flooding. At a lesser level spatial color dependence has also been found to exist for frontilluminated CCDs, although no time dependence is expected. Differences of $1 \%$ or so between the relative response across a front-illuminated CCD at 650 and $700 \mathrm{~nm}$ wavelength light have been seen in our laboratory. For back-illuminated CCDs, the problem is usually much worse and, in addition, interference fringes with amplitudes of $10 \%$ or more are often detectable at wavelengths about $800 \mathrm{~nm}$.

These effects mean that changes in the color of sky background, for example, may make background subtraction less accurate than it should be. They also may require careful calibration of each detector at each wavelength of interest. However, for time-dependent relative photometry in space, the CCD appears capable of a relative precision of 1 part in $10^{5}$ and is as close to an ideal detector as we are likely to see.

L.B.R. wishes to acknowledge support through Sverdrup Technology, NASA Grant No. NAS2-13769.

\section{REFERENCES}

Buffington, A., Hudson, H. S., and Booth, C. H. 1990, PASP, 102, 688

Buffington, A., Booth, C. H., and Hudson, H. S. 1991, PASP, 103, 685

Elliot, J. L., Dunham, E. W., Baron, R. L., Watts, A. W., Kruse, S. E., Rose, W. C., and Gillespie, C. M. 1989, PASP, 101, 737

Gilliland, R. L., and Brown, T. M. 1988, PASP, 100, 754

Gilliland, R. L., et al. 1991, AJ, 101, 541

Jorden, P. R., Deltorn, J. M., and Oates, A. P. 1994, SPIE, 2198

Young, A. T., et al. 1991, PASP, 103, 221 
